\title{
Role and Function of Endocannabinoid System in Major Depressive Disease
}

\author{
Erhan Yarar \\ Translational Medicine Expert (Member of the Cebede Cannabinoid Clinic Slovenia), Nutrigenetics and Epigenetics \\ Society (NES), Ankara, Turkey
}

\section{Keywords}

Endocannabinoid system · Endocannabinoids · Cannabidiol · Major depressive disease

\begin{abstract}
The endocannabinoid system (ECS) is a neuromodulator system with a crucial role in CNS and the reaction to endogenous and exogenous compounds and inflammation. Cannabidiol (CBD) is a basic part of the ECS which is the overwhelming causative and/or protective factor of major depressive disease (MDD). CBD interacts with brain-derived neurotropic factor (BDNF) that responds to inflammation, dysregulations of the hypothalamic-pituitary-adrenal (HPA) axis, and many more imbalances in MDD patients for which the ECS is a vital part to analyze, diagnose, and reflect the treatment. The ECS and MDD appear to have strong connections and interactions, so interest in ECS and CBD use in MDD patients is developing as a rescue resort. 02020 The Author(s).

Published by S. Karger AG, Basel
\end{abstract}

\section{Introduction}

The pathophysiology of major depressive disease (MDD) has been increasing clearly following the development of neuroscience and bioinformatics. It mainly involves these aspects: the dysfunction of the brain-related

karger@karger.com www.karger.com/mca

Karger"

GOPEN ACCESS
(C) 2020 The Author(s)

Published by S. Karger AG, Basel

This is an Open Access article licensed under the Creative Commons Attribution-NonCommercial-4.0 International License (CC BY-NC) (http://www.karger.com/Services/OpenAccessLicense), applicable to the online version of the article only. Usage and distribution for commercial purposes requires written permission. hormones, the hypothalamus-pituitary-adrenal (HPA) axis, the immune system, and the gut-brain axis (this latter parameter will not be dealt with in this review). It has obvious biological foundations, such as brain changes including unbalanced neurotransmitters, impaired neurogenesis, neuroplasticity decline, and abnormal neuronal circuitry. The most disturbing factor in MDD is it may or may not present relapses and/or remissions by several internal and external causes. Techniques and drugs come up short and so making the cannabidiol (CBD) an attractive and comfortable tool for acute and chronic treatment options. To overcome the grave consequences, many diverse targets should be considered such as mood-enhancing catecholamines which bring about progressively positive state of mind and antidepressants work to build them to a satisfying level but cannot succeed in preserving that level in long term after cessation and are accompanied by many side and adverse effects such as bone fractures, hyperlipidemia, suppression of bone marrow, and endocrinological, cardiological, and gastrointestinal issues, to mention a few, whereas CBD as observed balances catecholamines, acts on HPA axis dysregulation, reduces inflammations, regulates synaptic neuroplasticity, and impacts almost on all systems in the body in a positive manner making it attractive as a main and/or an adjuvant treatment option. Role and function of ECS and CBD in MDD and ECS's place in the brain of a person diagnosed with MDD should be brought to the attention 
of the academic circles. This mini review aims this and proposes that the ECS might open a new avenue to form a unifying theory for MDD in future studies. MDD by large is still an obscure phenomenon, and there are several theories in pursuit of explaining this by isolating other psychiatric diseases. Often these theories also come up short in identifying the role of CBD's role in these theories. ECS's place in MDD is not sufficiently explored in full extent in academic circles, but powerful indications show it might play a vital role in foreseeable future such as putting a unifying theory as ECS is interconnected with almost all cell communications in body and brain and MDD-related regions. This is of utmost importance because of adverse effects and insufficiency of conventional psychiatric drugs in treating MDD which also posit numerous obscure parameters, making $\mathrm{CBD}$ with its unique safety profile a reliable option. This review will look at the pathophysiological aspects of MDD, CBD's effect on MDD, and ECS's impact in these parameters. CBD's benefits are not ignorable for diverse diseases arising from MDD. It seems a promising candidate as an adjuvant treatment option along with conventional medications for MDD. It has negligible side effect profile and pleiotropic benefits serving not only the "body" but also the "soul."

\section{Role and Function of Cannabidiol in MDD}

The essential components of the endocannabinoids (ECs) are tetrahydrocannabinol (THC, a crystalline compound, i.e., the main active ingredient of cannabis; it is psychoactive and anxiogenic) and CBD (a naturally occurring component in the resinous flower of cannabis; it is antipsychotic and anxiolytic). Although a balanced ratio of $\mathrm{CBD} / \mathrm{THC}$ also brings benefits in MDD, this review focuses on CBD. The ECS is composed of the cannabinoid receptors $\mathrm{CB} 1$ and $\mathrm{CB} 2$, the $\mathrm{CB} 1$ and $\mathrm{CB} 2$ endogenous ligands, $\mathrm{N}$-arachidonoylethanolamine (AEA), and 2-arachidonoylglycerol (2-AG), a specific and not yet identified cellular uptake mechanism, and the enzymes for EC biosynthesis are $N$ acyl-phosphatidylethanolamine-selective phosphodiesterase or glycerophosphodiesterase $\mathrm{E} 1$ and diacylglycerol lipase $\alpha$ or $\beta$, or inactivation and those for AEA and 2-AG biosynthesis are fatty acid amide hydrolase (FAAH) and monoacylglycerol lipase (MAGL), respectively. The ECS components, arachidonate-based ECs, AEA (bliss molecule) and 2-AG play positive roles in MDD, whereas FAAH which clears AEA and 2-AG is a negative player, and inhibition of
FAAH is a vital part of treating MDD which is not a fact with standard medications and makes CBD's effect clear and indispensable. Cannabinoid receptors and their endogenous ligands are found all through the limbic system, where they modulate synaptic neurotransmission and influence MDD in a positive manner. A reduced ECS signaling might increment MDD side effects, so improving the signaling appears to be a novel alternative for the treatment of MDD. CBD promotes antidepressant-like effects by enhancing the monoaminergic neurotransmission, inducing hippo multiple neurogenesis, and normalizing the hyperactivity of HPA axis, thereby lessening and eventually eliminating MDD to an obvious extent.

The ECS is a complex system with additional components such as putative $\mathrm{CB} 1$ antagonist peptides such as hemopressins, peroxisome proliferator-activated receptor-a (PPAR-a), and its ligands, such as oleoylethanolamide (OEA) or palmitoylethanolamide (PEA) and $\mathrm{N}$ arachidonoyl-DA (NADA), which activates both TRPV1 and $\mathrm{CB} 1$ receptors (CB1R) [1]. Memory function is mainly related to hippocampus, motor to basal ganglia, and mood to prefrontal cortex and so any dysfunction of the ECS can be considered as a causative factor in MDD.

The CB1R is one of the foremost abundant G proteincoupled receptors (GPCRs) within the CNS and is found in especially high levels within the neocortex, hippocampus, basal ganglia, cerebellum, and brainstem [2]. CB1Rs are moreover found on peripheral nerve terminals and a few extraneural regions such as the testis, eye, vascular endothelium, and spleen.

CB1Rs are exceedingly improved at presynaptic and axonal compartments, confining their work to sites of synaptic action. In expansion to its area on the cell surface, intracellular localization of CB1Rs has moreover been detailed in heterologous systems. CB2 receptors are expressed at much lower levels in the CNS compared to $\mathrm{CB} 1$. CBD indirectly affects the CB1Rs by pausing the enzymatic breakdown of AEA, enhancing AEA, and permitting it to remain in the system longer and provide positive medical benefits in MDD. Any dysfunction in this system leads not only to MDD but also to other psychiatric disorders and also causes some pathological issues which is not a subject to discuss in this review. EC signaling is widely distributed throughout corticolimbic circuits that are linked to the stress response.

ECs are released from lipid precursors when needed and serve as reverse signaling messengers in GABAergic and glutamatergic synapses and modulators of postsynaptic transmission, interacting with other neurotransmitters including DA to which docking $\mathrm{CBD}$ has only mini- 
mal effect which is a negative fact for monoamines. AEA can be characterized as a stress-responsive neurotransmitter and is to a great extent not as tonically dynamic as $2-\mathrm{AG}$ within the adult brain. Be that as it may, in the event that AEA is initiated to be always dynamic, the brain will downregulate its responsiveness to AEA, in this way diminishing its neuromodulatory usefulness, and the signaling work carried out by stress-responsive varieties of AEA levels will be disturbed meaning progression of MDD symptoms.

AEA and 2-AG bind to a component of GPCRs, of which the cannabinoid $\mathrm{CB} 1 \mathrm{R}$ is deeply distributed in areas of the brain in charge of motor control, cognition, emotional responses, motivated behavior, and homeostasis. 2-AG and AEA are synthesized and degraded by FAAH, and CBD inhibits FAAH, thus increasing the AEA. Particular enzymatic pathways such as FAAH have physiological and pathophysiological parts to these 2 ECs by expanding or halting the MDD indications and preventing them from emerging as a disease. AEA is synthesized within the brain, where levels are controlled by firmly directed degradation pathways. Its impacts within the brain are greatly localized. Like 2-AG, it is a retrograde neurotransmitter whose release can influence numerous cells within the neighboring synaptic space, but not past that space. It does not have the diffuse activity of a hormone; its release is locally controlled and does not influence the organism systemically within the way that exogenous cannabinoid organization does. AEA protects neurons.

AEA re-establishes the raised $\mathrm{CB} 1$ expression conjointly to promote the diminished level of 5-HT within the hippocampus. In expansion, AEA re-establishes the elevated NA levels within the prefrontal cortex and actuates a neuroprotective impact by re-establishing the diminished glutathione and expanded lipid hydroperoxide levels together with the diminished superoxide dismutase movement watched in the hippocampus and/or prefrontal cortex which zone significant brain regions included within the control of temperament, aggression and/or impulsivity, and decision-making, which are unquestionably changed in MDD; all these demonstrate reversed MDD indications.

CBD is responsible for a sharp inhibition of neuronal activity, which is required to maintain complex firing patterns across widespread brain networks. Having such a fundamental role, it is no surprise that malfunctions in this system are associated with a myriad of brain disorders such as MDD including anxiety.

CBD treatment interacts in a positive manner with AEA. Outside the brain, the EC is one of the critical mod- ulators of the autonomic nervous system, the immune system, and microcirculation when dysregulated causing MDD symptoms. Altered EC function causes pathophysiological aspects of MDD and related suicidal behavior [3]. CB1 and CB2 are transmembrane GPCRs located in the central and the peripheral nervous system. The most abundant and causative cannabinoid receptor is the CB1 cannabinoid receptor, but $\mathrm{CB} 2$ cannabinoid receptors, transient receptor potential (TRP) channels, and PPARs are also involved by some cannabinoids.

As exogenous cannabinoid CBD interacts with 2-AG and AEA and brings out its effects in MDD. MDD is also a disease of imbalances and dysregulation in the structural and neuronal/nervous system. CBD inhibits EC reuptake, activates transient receptor potential vanilloid 1 and GPCR 55, and enhances the activity of 5-HT1A receptors. As an essential part, endogenous cannabinoid AEA acts as a partial agonist at CB1Rs. CBD acts as an agonist at 5-HT1A receptors [4] and so works as a fastacting antidepressant and anxiolytic medication [5]. A reduced affinity of 5-HT1A receptors is identified in the brains of MDD individuals [6]. As a 5-HT1A serotonergic agonist and activator of the endogenous ECS, CBD is an antagonist at GPR55 receptors and an activator of transient receptor potential channels [7]. CBD's activity at 5-HT1A receptors seems to drive its neuroprotective, antidepressive, and anxiolytic benefits, although the mechanism of action how CBD diminishes and eliminates the anxiety is still unclear. This is imperative as these receptors help in releasing brain-derived neurotrophic factor (BDNF) and are responsible for neuroprotective impacts of the 5-HT receptors, and these exceptionally receptors are too related to the neurobiology and etiology of MDD. The mechanisms how 5-HT1A receptors adjust to stress and initiate antidepressant-like impacts are also not clear. Stressful events and insults impede postsynaptic 5-HT1 Amediated effects in limbic regions, in hippocampus, and cause individuals incapable of adjusting themselves to stress-induced behavioral results all aroused by MDD so bringing out to facit circles.

The BDNF is a protein located and manufactured primarily within the brain and spinal cord which fortifies the vitality of nerve cells and induces the growth, maturation, and maintenance of them. It is active at synapses where cell-to-cell communication occurs, which adjusts in reaction to events, a characteristic called synaptic plasticity (SP), so any impairments in SP apparatus contribute to psychiatric disorders. BDNF protein helps regulate SP. Evidence demonstrates that BDNF levels are decreased in $\mathrm{MDD}$. Increased levels of BDNF seem to contribute to 
improving MDD symptoms. CBD brings BDNF to a desirable level intensely and keeps this adjusted in longterm application by progressing and treating MDD symptoms.

CBD invigorates in acute stages fast and in chronic stages maintains antidepressant-like impact relevant for MDD. These impacts are related to swift changes in SP within the medial prefrontal cortex (mPFC) through activation of the BDNF-TrkB signaling pathway. CBD can also act specifically on gamma aminobutyric acid (GABA) receptors which is one of the essential neurotransmitters of the brain related to MDD.

When a neuron is under extraordinary inhibition due to GABA action, it can synthesize 2-AG and AEA and send them to the previous neuron whereupon they dock to $\mathrm{CB} 1$ and $\mathrm{CB} 2$ receptors, driving to a cascade of molecular responses that eventually diminishes GABA release. The diverse capacities of GABA are determined by the area of its receptors as well as their particular structure. Each GABA receptor is composed of 5 proteins drawn from a pool of 19 potential proteins, with diverse arrangements determining what substances can enact them and how they work with respect to potency, speed, and duration of activity. Recently, it has been found that the ECs can too act specifically on GABA receptors in a way that is exceptionally similar to how drugs such as benzodiazepines and barbiturates work. It is well possible that such a direct modulation of GABA receptors contributes to the restorative impacts of CBD observed in MDD. $\mathrm{CBD}$ and related compounds including particular terpenes offer promising treatment options for MDD. Proof of the part of the ECS within the neurobiology of neuropsychiatric disorders is starting to rise. The behavioral impacts of ECs are mediated through the central cannabinoid CB1R. Imbalanced ECs, and the density and coupling efficacy of CB1Rs, have been reported within the prefrontal cortex of MDD patients. ECS is a ubiquitous lipid signaling system and has vital administrative capacities all through the body including the innate and immune system. According to studies, forebrain 5-HT1A receptors are primary targets of antidepressant drugs. The mechanism is still open to debate, but these receptors interfere in neuroplastic changes driving to neurogenesis (Samuels et al.). Antidepressant treatment may increase hippocampal neurogenesis but once more with undesirable negative impacts where CBD effect appears with nearly null side impact. CBD's antidepressant impacts mediated by help of 5-HT1A receptor-mediated neurotransmission [8] and its anxiolytic activity in connection to MDD in details are yet to be decided.

\section{Cannabidiol's Place in MDD and Related Brain Regions}

The prefrontal cortex, hippocampus, and amygdala play an imperative role in the control of emotion, stress reactions, self-control, and cognitive response, but in MDD patients the function of the prefrontal cortex and hippocampus is impaired, whereas the movement of the amygdala is expanded. In this manner, long-lasting antidepressant treatments point to extend the neurotrophic variables, fortify neurogenesis, diminish the hippocampus neuronal apoptosis, and progress the mood and cognition; however, none of them suffice to prevent the relapses and amplifying the remission periods of MDD with side impacts; in this manner, CBD's impact with neglectable side effects is shown multifaceted and with self-evident success.

ECs such as AEA and 2-2-AG act by activating cannabinoid type 1 (CB1) or 2 (CB2) receptors. The anxiolytic effect of medications such as CBD initiates EC's impacts on increase in AEA levels in several encephalic regions, including the prefrontal cortex, so activation of CB1 receptors by CB1 agonists infused directly into these regions is usually anxiolytic.

In any case, depending on the encephalic locale being investigated and on the stressful events, reverse impacts are observed, as detailed interior the ventral hippocampus relates to stress, feeling, and influence. Anxiolytic impacts of CBD in models of MDD are associated to specific receptor components and brain districts. The midbrain dorsal periaqueductal gray (PAG) is integral to anxiety, organizing autonomic and behavioral reactions to threat, and PAG prompting in humans produces thoughts of distress and fear. Just to name, this region shows how deeply MDD affects a person's life because PAG is an anatomic and functional interface between the forebrain and the lower brainstem and incorporates a major part in integrated behavioral reactions to internal (e.g., torment) or external threat stressors. The PAG comprises distinct columns that get specific inputs from the prefrontal cortex, amygdala, hypothalamus, and nociceptive pathways and any disorder facilitates symptoms of MDD. By means of its associations with diverse brainstem nuclei, the PAG facilitates particular patterns of cardiovascular, respiratory, motor, and pain modulatory reactions. These reactions shift according to the sort of stress and the subject's perception of the threatening stimulus which is positively altered by introduction of CBS.
Med Cannabis Cannabinoids 2021;4:1-12 DOI: $10.1159 / 000511979$
Yarar 
With regard to fear, CBD plays its part in different components of the brain, as well as the neural circuitry supporting fear memory handling comprises other interconnected zones such as the hippocampus, amygdala, and PAG. Infusing CBD into the PAG diminishes anxiety in paradigms assessing intrinsic fear. In any case, this zone is additionally imperative for intervening freezing and other protective behaviors in reaction to learned threats, proposing that $\mathrm{CBD}$ regulation of fear memory expression may include the PAG. In humans, $\mathrm{CBD}$ decreases anxiety and autonomic excitement amid the seeing of fearful facial expressions, which is accompanied by diminished activity and functional connectivity between the amygdala and mPFC. In mice, $\mathrm{CBD}$ diminishes amygdala activation as measured by c-Fos expression. This raises the plausibility that the amygdala plays a part in mediating CBD control of fear memory.

CBD also decreases mPFC-hippocampus functional connectivity amid cognitive processing. This proposes that the hippocampus may moreover be involved in CBD regulation of certain aspects of learned fear. After termination, fear expression is low when tried within the extinction setting, but fear renewal happens exterior of this context. This relevant control of fear termination includes the hippocampus and its associations with the $\mathrm{mPFC}$ and amygdala. CBD might decrease fear relapse and/or the spontaneous recovery of fear that happens over time after termination through its activities on the hippocampus-mPFC-amygdala circuit. CBD modulation of this circuitry may also regulate learned fear in other paradigms with translational significance. CBD moreover diminishes functional connectivity between the amygdala and anterior cingulate cortex (ACC), a brain region where both affective and cognitive information converge, pointing to both an anxiolytic effect of CBD and a critical modulatory role of the ACC which apart from the emotion-regulating properties of $\mathrm{CBD}$, the CBD-ACC relationship has not been systematically investigated too. EC signaling is broadly dispersed all through corticolimbic circuits that are connected to the stress reaction. Distinctive scanning procedures show that AEA and 2-AG are synthesized within the hypothalamus, amygdala, hippocampus, and prefrontal cortex [9]. Autoradiography of cannabinoid receptors in brain areas from a number of mammalian species, including human, reveals a unique and preserved conveyance; binding is most dense in surge nuclei of the basal ganglia - the substantia nigra pars reticulata and globus pallidus - and inside the hippocampus and cerebellum [10]. The exact im- portance of these volumetric varieties from the norm is still obscure.

MDD appears to lie in various brain areas as well as no place in particular. A number of clarifications can be deciphered and have been proposed with regard to this state of affairs. One plausibility is that MDD is localized in numerous brain regions at the same time. This may emerge from a common molecular peculiarity inside the neurons of multiple brain regions. Receptor variations from the norm or varieties from the standard in signal transduction might be such conceivable anomalies. The main subcortical limbic brain districts involved in MDD are the amygdala, hippocampus, and the dorsomedial thalamus, and gene expression in the dorsal hippocampus correlates with cortical regions involved in information processing, while genes expressed in the ventral hippocampus correlate with regions involved in emotion and stress (amygdala and hypothalamus) [11] all docked to the ECS, and CBD acts on all of them via epigenetic regulation.

Both structural and functional anomalies in these regions have been found in MDD. Decreased hippocampal volumes and amygdala have been noted in subjects with MDD. CBD moreover attenuates blood-oxygen-level-dependent activation within the left amygdala, and the front and posterior cingulate cortex in reaction to escalation frightful faces, and diminishes amplitude in skin conductance fluctuation, which is exceedingly connected with amygdala activation. Subjects who remit with treatment have indeed been appeared to have bigger pretreatment hippocampal volumes, whereas those with smaller hippocampal volumes were reported to be more inclined to relapse. Diminished amygdala core volume has been reported in MDD, but CBD is shown to have no impact on gray matter volume and cortical thickness. Short-term exposure to exceedingly purified CBD does not influence cortical macrostructure but re-establishes hippocampal and amygdala volume loss, and in fact most MDD patients have diminished size of amygdala as shown in MRI (personal record).

CBD decreases the already reduced hippocampal neurogenesis and dendrite spine thickness initiated by consistent stress and avoids microglia activation and the diminish within the number of parvalbumin-positive GABA neurons MDD and equalizations cell destiny regulatory pathways such as autophagy and other essential pathways for neuronal survival and so advancing the obliterating results related with neurodegeneration [12]. In spite of the fact that mood disorders are not typical neurodegenerative disease, neural progression, diminished neurogenesis, and neuroplasticity, inflammation, 
cell death, and dysregulation of monoamines occur in a few patients with MDD.

An imbalance inside the fundamental excitatory (glutamate) and inhibitory (GABA) neurotransmitter systems inside the brain plays a part within the pathophysiology of MDD. Subsequently, the ECS appear to represent a therapeutic target for $\mathrm{MDD}$ as a regulator of glutamate and GABA release by means of the CB1R. CBD can re-establish cannabinoid/GABAergic signaling deficits in regions of the brain implicated in pathophysiology of MDD. Also, CBD increases mTOR signaling in rat models of neurodegeneration which is an additional burden on MDD patients. It is still obscure whether these instruments participate in CBD-induced antidepressant effects. All these processes and effects are still open to talk about and require more studies.

\section{Theories on Pathophysiology of MDD and CBDs' Antidepressant Mechanism}

The ECS is highly involved in MDD, and CBD has several pleiotropic roles in all of them as a regulating factor and explains to a great extent the causes of MDD and the application of CBD affecting the ECS. There are some pathophysiological speculations how really MDD as a disease emerges. These theories are actually interconnected with an obvious need for a unifying theory. In fact the logical way is considering all of them in identifying the MDD. One by one and derived from different approaches, these theories along with other minor ones come up very short in explaining the etiology of MDD, but they may reflect diverse spectrums of MDD, and it is worth to sum up them here with their relation to the ECS and CBD.

\section{Monoamine Theory and Cannabidiol}

Monoamine theory depicts that MDD is an aftereffect of a distorted monoamine synapse framework, especially serotonin (5-HT), noradrenaline (NA), norepinephrine $(\mathrm{NE})$, and dopamine (DA), lowering extracellular monoamine complexes and neurotransmission, and suggests that the basis of depression is a reduction in the levels of $5-\mathrm{HT}, \mathrm{DA}$, and NE in the body. The ECS is highly interconnected with the monoamine neurotransmitters. In fact, one study indicates that CBD reduces DA level without affecting hypothalamus and increases 5-HT level in the hypothalamus stimulating neurotransmitter synthesis. CBD reduces hypothalamic NE synthesis and DA re- lease and inhibits 5-HT synthesis, thereby showing its anxiolytic role [13]. CBD's anxiolytic role is dependent on brain 5-HT levels but not NA, so CBD can exert its effect on catecholamines indirectly. It is clinically active only as a neglectable and partial agonist at the DA D2 receptors.

In addition to binding to $\mathrm{CB} 1$ and $\mathrm{CB} 2$ receptors of the ECS, there is proof that CBD sanctions 5-HT1A serotonergic and TRPV1-2 vanilloid receptors, antagonizes alpha- 1 adrenergic and $\mu$-opioid receptors, represses synaptosomal take-up of NA, DA, 5-HT, and gammaminobutyric acid and cellular take-up of AEA, acts on mitochondria $\mathrm{Ca} 2$ stores, blocks low-voltage-activated (T-type) $\mathrm{Ca} 2$ channels, invigorates activity of the inhibitory glycine-receptor, and restrains activity of FAAH, and BDNF-TrkB interactions may modulate catecholamine release from adrenal chromaffin cells under acute stress conditions. There are also evidences of CBD's modulatory effects on DA activity within the mesolimbic pathway, functional interactions with the 5-HT receptor system, and their downstream molecular signaling effects relieve the symptoms in MDD; the inhibitory action of CBD on catecholamine secretion at the level of the adrenal medulla is clearly observed [14]. With this context, $\mathrm{CBD}$ improves and restores 5-HT and BDNF levels via 5-HT1A receptor activation and restoring 5-HT levels. There are CB1Rs located in hippocampus and amygdala, and any stress and anxiety is a result of the bed nucleus of the stria terminalis coordinating a stress-based response in conjunction with the amygdala which affects catecholamines. CBD produces its anxiolytic effects via activation of the 5-HT1A receptor associated with 5-HT, affecting positively the amygdala. Studies show that CBD acts via the modulation of the 5-HT1A receptors [15].

The acute anxiolytic and antidepressive activities of intense CBD are proposed to be intervened by 5-HT1A receptors. The crosstalk among cannabinoids and serotoninergic signaling is unpredictable. In rodents, CBD administration into the dorsal parcels of PAG produces against aversive impacts in the elevated plus maze and flight-induced by local electric stimulation [16]. Other cerebrum regions such as the basal ganglia, the bed nucleus of stria terminalis, the prelimbic PFC [17], and the dorsal raphe core likewise appear to mediate $\mathrm{CBD}$ impacts through 5-HT1A receptors.

The molecular mechanism by which CBD encourages 5-HT1A receptor activation is still obscure. Evidence proposes that it might include allosteric regulation of this receptor, advancing 5-HT1A agonist-related stimulation of [35S]GTPS binding [18] increment in 5-HT release or potentially reuptake inhibition or the circuitous develop- 
ment of heterodimers comprising 5-HT1A and different receptors, for example, CB1. NE plays a role in stress reactions and the control of feelings. NE levels have been found to be low in conditions of MDD. NE enacts the amygdala. The amygdala can also improve long-term capacity of stressful memories within the hippocampus and striatum. Cannabinoids repress epinephrine (adrenaline) secretion in rabbit isolated adrenal organs. Since adrenaline in plasma starts solely from adrenal chromaffin cells, impacts of cannabinoids on adrenaline release were considered in isolated adrenal organs. In vivo catecholamine exocytosis from chromaffin cells is controlled by the activity of presynaptic splanchnic nerves innervating the adrenal medulla. Incitement of the nerve terminals causes release of acetylcholine and noncholinergic neurotransmitters (e.g., opioid peptides and vasoactive intestinal peptide), which at that point enact postsynaptic receptors to evoke catecholamine secretion. With regard to stress induced by epinephrine, cannabinoids brings down the electrically evoked adrenaline release in rabbit isolated adrenal glands; the likely component could be a presynaptic CB1R-mediated inhibition of acetylcholine release from preganglionic sympathetic neurons innervating the adrenal medulla. This diminishment in adrenaline release most likely accounts for the marked decrease in plasma adrenaline concentration and reduced stress observed after cannabinoid administration within the pithed rabbit [19] which offers CBD as an alternative to treat MDD patients suffering from extensive release of epinephrine causing stress with no motive either.

ECs and their receptors, basically the CB1R type, work as a retrograde signaling system in numerous neural connections inside the CNS, especially in GABAergic and glutamatergic synapses. They moreover play a modulatory work on DA transmission, in spite of the fact that CB1Rs do not show up to be found in dopaminergic terminals, at least within the major brain districts receiving dopaminergic innervation, for example, the caudate-putamen and the nucleus accumbens/prefrontal cortex. In this manner, the impacts of cannabinoids on DA transmission and DA-related behaviors are by and large indirect and exerted through the balance of GABA and glutamate inputs received by dopaminergic neurons [20].

Although cannabinoid CB1Rs are densely expressed in neurons expressing DA D1 receptors (D1Rs), it is not completely understood to what degree they modulate emotional behaviors. Cannabinoid-induced increments in nucleus accumbens DA concentration are thought to emerge from an increase within the mean firing rate of DA neurons inside the ventral tegmental range. Dopami-

Role and Function of ECS in MDD nergic neurons of the midbrain are the most source of DA inside the mammalian CNS. Their loss is related with one of the first recognizable human neurological disorders. Dopaminergic neurons are found within the substantia nigra pars compacta. In spite of the fact that their numbers are few, these dopaminergic neurons play a crucial role inside the control of various brain capacities including intentional movement and a wide cluster of behavioral forms such as mood and stress. Another point is that decision-making which is an ethologically adaptive component that is impeded in MDD. Action inside the mesocorticolimbic DA system has been traditionally related with decision-making. The ECS through its actions on inhibitory and excitatory synapses modulates DA activity and decision-making. Cannabinoid-DA interactions pay emphasis in 2 forms where DA has been proposed as a key neurotransmitter, such as the basal ganglia function and the corticolimbic processes. Dysregulation or overactivity of DA transmission where CBD might be of therapeutic potential for MDD conceivably through activities that facilitate, among others, a normalization of DA transmission [21] is apparent.

The diminishing in monoamine neurotransmission results either from too little synapses released by presynaptic neurons, or from quickly reabsorbed/manipulated synapses or a lessening in postsynaptic receptors but also observed in other psychiatric conditions not making it a sole player in MDD. Studies have conflicting results, and despite any positive observations, monoamine theory is still being very much open to debate.

\section{Dysregulation of HPA Axis Theory and Cannabidiol}

The HPA axis could be a crucial portion of the stress reaction system, and dysfunction of the HPA axis is one of the foremost imperative mechanisms behind MDD. Both mental and physiological stresses enact the HPA axis and invigorate the release of corticotrophin-releasing factor (CRF) and vasopressin (AVP) by the hypothalamus. Both CRF and AVP start the anterior pituitary organ to release adrenocorticotrophic hormone (ACTH), which progresses the release of adrenocortical hormones, including glucocorticoid (GC), and causes an increase in circulatory GC levels, which controls the secretion of CRF and AVP by the hypothalamus, forming a negative feedback circuit. In any case, over half of the MDD patients show negative feedback dysfunction of the HPA axis, including a constant increase in circulatory GC and $\mathrm{ACTH}$, and some of the patients in fact endure from hypercortisolemia. Cortisol is the stress 
hormone, and it lies at the crossing point of our stress response and anxiety. Stress leads to damage or inhibition of neurogenesis through hypercortisolemia, reduced BDNF, or extended glutamate. Cortisol seems to form a domino effect that hardwires pathways between the hippocampus and the amygdala in a way that might make an appalling cycle by making a brain that gets to be inclined to be in a consistent state of fight-or-flight. In case of intense or unexpected stressful situations, the ECS works to counter the spike in cortisol in arrange to bring the system back to adjust. It is persistent that cortisol and stress definitely deplete this system. Chronically raised cortisol can cause brain damage. This damage can result in anxiety an additional negativity figure of MDD.

CBD diminishes cortisol levels, supports stress response framework (AEA), and energizes brain repair by means of neurogenesis, and this comes about in a coordinate impact on uneasiness both brief and long term. In spite of the exceptionally strong preclinical and clinical data of a dysregulation of the HPA axis in stress-related mental disorders, such as MDD, no drug has been approved that targets particular components of the HPA axis. In extension, no test is routinely utilized interior the clinical setting to recognize patients with measureable HPA axis anomalies. In fact, there is proof that not all MDD patients show alterations of the HPA axis and subsequently not all of them would benefit of a very particular treatment, centering on only HPA axis components. Major modifications of the HPA axis system that can be exchanged by antidepressant treatment are regularly seen in MDD patients. Persuasive proof focuses on the incorporation of a dysfunctional glucocorticoid receptor (GR) system in these changes. Support for this also comes from studies of transgenic mice that express an antisense RNA, complementary to the GR mRNA, and have different neuroendocrine characteristics of human depression as well as altered behavior. Several of these neuroendocrine and behavioral characteristics of the transgenic mice can be exchanged by antidepressants. A conceivable clarification for this may be that the antidepressant-induced increase in GRs renders the HPA axis touchier to glucocorticoid feedback. The GR plays an important role in the HPA axis function during depression; the excessive circulating GC reduces the sensitivity of GR, while antidepressant therapies increase the GR expression, enhance the GR function, and improve the negative feedback medicated by GR. Later research found that HPA axis dysfunction also reduces BDNF expression [22], inhibits 5-HT synthesis, decreases Glu receptor expression, and even disturbs neuroplasticity and neural circuits.
The amygdala invigorates the nerve center which thusly energizes the HPA axis which by expanding blood cortisol complexes follows up on a positive way component to influence the amygdala. Overexcitation of the HPA axis is prevented with a negative examination instrument by the hippocampus which limits the HPA axis action dysfunction. Studies show raised cortisol, ACTH, and corticotrophin releasing hormone (CRH) levels in patients with MDD and raised glucocorticoid complexes appear to cause restraint of average prefrontal cortex excitation in MDD. Low blood BDNF levels are observed altogether in MDD. CBD controls acute stress HPA actuation impacting 5-HTR1A receptors. Constant stress and inflammation cause both the HPA axis and ECS dysfunction. It is critical to address the EC deficiency and rebalance the HPA axis and increase the BDNF levels.

The ECS can be rectified by bringing down $\mathrm{CRH}$ and FAAH with exogenous CBD, thereby raising AEA and lowering glutamate. Expanded levels of AEA are specifically correlated with improved wellness, mood, and stress reaction and so making CBD an excellent tool to boost EC signaling and progress the regulation of the HPA axis. CBD actually increases ECS tone which also helps to improve the control of homeostasis over the HPA axis. It is critical to reestablish the EC deficiency and rebalance the HPA axis to which CBD shows its positive impacts by raising $\mathrm{AEA}$ and bringing down glutamate. Expanded levels of AEA are specifically related with improved mood and so reducing negative shape of MDD by bringing down cortisol and CRH levels.

Diminishment of AEA-CB1R signaling inside the amygdala intervenes the anxiogenic impacts of $\mathrm{CRH}$, and $\mathrm{CB} 1 \mathrm{R}$ activation is fundamental to negative feedback of the neuroendocrine stress response and secures against the opposing impacts of chronic stress; chronic stress disables EC signaling inside the hippocampus and amygdala, driving to anxiety, and individuals with MDD show up elevated CB1R and diminished peripheral AEA, suggestive of diminished EC tone.

The ability of CBD to regulate acute stress HPA activation might be explained, at least in part, by its action on 5-HTR1A receptors. CBD regulates the HPA axis responses to stress and impairment of EC signaling in the hippocampus following acute/chronic stress could contribute to the development of MDD. Finding new EC molecular targets to modulate the "stressed hippocampus," therefore could be a helpful contribution for novel therapeutic approaches, yet there are no drugs available so far making CBD again a reliable medication. Although several studies indicate a strong connection in between MDD 
and HPA axis dysfunctions, this theory of HPA axis is also open to debate and cannot be considered as a sole contender.

\section{The Inflammatory Hypothesis of MDD and Cannabidiol}

One other essential approach to MDD is the inflammatory hypothesis which posits that immune hyperactivation and dysregulated cytokine production are involved in MDD. Cytokines are cell signal transducing proteins or polypeptides that intervene and direct immune reactions and inflammation. They cross the blood-brain barrier, in this manner forming numerous perspectives of MDD pathophysiology, including enteric and general neurotransmitter system, neuroendocrine function, and neural plasticity. Be that as it may, there is clear proof that changes in cytokine levels are not reliably related with positive results amid MDD treatment.

Inflammatory hypothesis sets that in MDD the proinflammatory cytokines, including IL- 6 and TNF- $\alpha$, increase in sum, whereas the anti-inflammatory cytokines, including interleukin-10 (IL-10) and transforming growth factor-beta (TGF- 3 ), diminish, making the holistic resistant response tend to inflammation. The excessive proinflammatory cytokines repress the negative feedback of the HPA axis, increase the permeability of the bloodbrain barrier, decrease the synthesis of 5-HT, disturb the glutamatergic frameworks, and result in depression.

The proinflammatory cytokines, in expansion to actuating the HPA axis and in this manner expanding cortisol synthesis, also enact the tryptophan-kynurenine pathway. This comes about within the synthesis of the neurotoxic $N$-methyl-D-aspartate (NMDA) glutamate agonist quinolinic acid and 3-hydroxykynurenine subsequently improving oxidative stress and contributes to neurodegeneration which characterizes MDD. While antidepressants attenuate some of the endocrine and immune changes caused by inflammation, not all therapeutically effective antidepressants do so. This suggests that drugs which particularly target the immune, endocrine, and neurotransmitter systems may be more compelling than ordinary antidepressants which opens a new avenue for CBD. The blood levels of sIL-2R, TNF- $\alpha$, and IL- 6 have been found high in MDD has moreover been affirmed by a meta-analysis [23]. Decreased degrees of interleukins in MDD such as beneficial IL-10 are also questioned. Elevated CRP and IL- 6 are a part of MDD, and MDD was related with higher CRP levels in MDD [24]. Similarly, higher CRP and IL-6 levels are related with psychological indications of MDD.

Inflammatory markers do decrease BDNF articulation, yet in resistant MDD patients, both IL- $1 ß$ plasma concentrations are increased making them as partial yet effective candidates to identifying MDD. It is obvious that cytokines induce behavioral effects by activating inflammatory signaling pathways in the brain, leading to the reduction of growth factors such as BDNF, and the expression of these growth factors is affected by immune cells and the immune factors they secrete.

The treatment resistance is also a part of inflammation and several markers are unfortunately neglected in psychiatry. CBD seems to eliminate proinflammatory cytokines such as IL-1, IL-6, and interferon (IFN), and NF-KB pathway all causing to trigger the proinflammatory genes. CBD upregulates the STAT3 and restores homeostatic mechanisms. Altered BDNF function is involved in the structural changes and possibly impaired neurogenesis, neurotoxicity, and neuroinflammation in the brain of MDD patients showing a significant relationship between failed treatments and TNF, soluble TNF receptor 2 (sTNF-R2), and IL-6. Checking inflammatory markers and targeting inflammation or its downstream mediators via diverse treatment options are relevant for MDD patients.

CBD can re-establish cannabinoid/GABAergic signaling deficits in regions of the brain implicated in MDD. CBD enhances 5-HT and glutamate levels in the prefrontal cortex. 5-HT1A receptor mediates CBD-induced antidepressant-like impacts and increase of 5-HT/glutamate levels (Linge et al. [5]). One of the basic roles of CBD is that it diminishes inflammation within the central region of the brain and decreases levels of proinflammatory cytokines within the hippocampus (TNF- $\alpha$ ) and prefrontal cortex (IL-6). Inflammation and moreover infection diminish BDNF gene expression and so CBD increases BDNF by diminishing inflammation within the brain with its anti-inflammatory activity which is exceptionally critical figure that standard medicines can do it only to a minimum degree [25].

CBD plays a basic part in eliminating the inflammation as seen in serum/plasma inflammatory markers. The ECS has been shown both in vivo and in vitro to be included in directing the immune system through its immunomodulatory properties. CBD works by binding several subtypes of cannabinoid receptor within the brain and other organs. In expansion, the body produces ECs that moreover work through binding these receptors. Compounds that are chemically related to $\mathrm{CBD}$ have also 
been shown to function by binding other sorts of receptor, such as the NMDA receptor and the PPAR, or by affecting other cellular components, such as lipid rafts.

CBD and related compounds have been shown to either suppress or increase the production of pro-inflammatory cytokines such as TNF- $\alpha$, IL-1ß, and IL- 6 in both patients and animal models, indicating that these drugs can modulate proinflammatory mediators. Depending on the model system, the effects of these drugs do not always depend on their interaction with cannabinoid receptors.

$\mathrm{CBD}$ disposits the immune response away from $\mathrm{T}$ helper 1 (TH1)-cell responses by mechanisms that involve cannabinoid receptors. It is possible that signaling through these receptors, expressed by T cells, B cells, or antigen-presenting cells, suppresses the expression of TH1-cell-promoting cytokines and increases the expression of TH2-cell-promoting cytokines.

CBD regulates some of the inflammatory aspects of brain injury, through both cannabinoid-receptor-mediated and non-cannabinoid-receptor-mediated mechanisms. It is conceivable that ECs decrease brain edema and other aspects of neuroinflammation by inhibiting NMDA receptors, by functioning as antioxidants, and by decreasing the levels of proinflammatory cytokines within the brain. CBD suppresses inflammatory reaction and in this way attenuates disease symptoms. This property of $\mathrm{CBD}$ is intervened through numerous pathways such as induction of apoptosis in activated resistant cells, concealment of cytokines and chemokines at inflammatory sites, and upregulation of FoxP3+ regulatory T cells [26], including bipolar disorder, anxiety disorders, identity disorders, and schizophrenia. These data propose that inflammation is transdiagnostic in nature, happening in subpopulations of patients inside a number of psychiatric disorders and so making this hypothesis not a sole candidate for clarifying completely the MDD.

\section{Neuroplasticity Theory of MDD}

The recently emerged neuroplasticity theory of MDD is additionally an approach to clarify the pathogenesis of MDD. Neuroplasticity is considered to be one of the imperative characteristics of the nervous tissue which shows reversible changes which is called functional plasticity. It appears to modulate the expression of genotype into phenotype by persistent adjustment and thus bring around long-lasting impacts which is fundamental for MDD.
This hypothesis sets that depressive indications emerge from the impaired neuroplasticity, which can be initiated by numerous risky components, including neurotransmitter imbalance and insufficient BDNF. MDD patients not only show impeded neurogenesis but also present disturbed neuronal growth, decreased synaptic plasticity, impaired myelin function, and a decrease in total neuroplasticity [27]. CBD's impacts of plasticity can cause either positive or negative changes amid developmental plasticity, receptive plasticity, adaptational plasticity, and amid primary or auxiliary recuperation of damaged neuronal circuits, reparation plasticity. CBD in conjunction with neuroplastic mechanisms help repair and reestablish the neuronal circuitry damaged by MDD.

As CBD is appeared to upgrade BDNF expression, it indirectly leads to form new connections by enacting the BDNF-TrkB signaling pathway. Since CBD's affect is blocked when BDNF signaling is hindered within the brain, CBD starts to set up quick neurochemical and neuroplastic effects in limbic brain regions favoring stress coping strategies and resilience to MDD improvement. This theory is promising in all sense yet as neuroplasticity evolves with other components of abovementioned theories it is also not capable to fully explain MDD by isolating other neurological and psychiatric disorders and diseases.

\section{Discussion}

CBD has a complex pharmacology, and it interacts with multiple neurotransmitter systems involved in MDD such as serotonergic, glutamatergic, and EC systems. $\mathrm{CBD}$ induces cellular and molecular changes in brain regions related to MDD neurobiology, such as increased BDNF levels and synaptogenesis in the MPFC, as well as it increases neurogenesis in the hippocampus. Although all factors related to the abovementioned theories are considered playing roles in MDD, none of them could be accepted as sole candidates and there is no "unified theory" to explain the cause of the MDD, but almost all studies underline the effects of CBD and indicate CBD as a safe option for applying as alone or in combination with conventional drugs as an adjuvant medication in MDD.

CBD responds to several receptors known to regulate fear and anxiety-related behaviors, specifically the CB1R, the 5-HT1A receptor, and the (TRPV1 receptor. In addition, CBD may also regulate, directly or indirectly, the PPAR- $\gamma$, the orphan GPCR 55, the equilibrative nucleoside transporter, the adenosine transporter, additional 
TRP channels, and glycine receptors. Within this respect, CBD regulates the needed catecholamines, anti-inflammatory markers, and homeostasis too.

Despite the fact that reviews have exhibited the quieting, calming, loosening up, and anti-inflammatory impacts of CBD, clinical information from real and human cases is insignificant. ECS's relation with MDD and other points should be explored in extensive studies.

The anxiolytic effects of CBD present a bell-shaped dose-response curve, with anxiolytic effects observed at moderate but not higher doses. Preclinical evidence conclusively demonstrates CBD's efficacy in reducing anxiety behaviors in MDD. Current preclinical and human findings mostly involve acute CBD dosing in healthy subjects, so further studies are required to establish whether chronic dosing of CBD has similar effects in relevant clinical populations.

\section{Conclusion}

The principle finding of studies and practices is that CBD alongside conventional prescriptions and enhancements can be a successful compound to diminish MDD and other related issues as showed in a critical or analytical summing up especially of a medical case history of several patients. Further investigation should be directed to decide the permanency of patient's certain practices and to what extent they should keep taking the CBD. As CBD interacts with BDNF in a positive manner, it is highly possible a resilience to MDD symptoms and maybe a long-lasting healing. Since CBD also possesses anti-inflammatory properties, it directly hits the immune system anomalies, thereby contributing also to improvements.

Although human trials on CBD and ECS with respect to MDD are still not satisfying, observational data indicate a need to increase them. It is a solid fact that CBD is used among patients, and therefore physicians should not disregard patients' interest in CBD as a treatment option and continue to enlighten both patients and themselves about alternative therapies with the assistance and guidance of translational medicine experts. Combinations of $\mathrm{CBD}$ and conventional medications are of utmost importance and needed as necessary as patient's unique medical conditions such as endocrinology, cardiology, and gastroenterology. The ECS's vital role in MDD still needs extensive studies.

There are basic and emerging hypotheses about how MDD develops, but none of them suffice to clarify this problematic to a satisfying degree. This phenomenon is open to debate, and possibly it is time to consider that there is no academic need for persistency for each one and a translational approach is the solution which is of utmost importance. A scientific and sound need for all of these theories ought to be utilized to illuminate different perspectives of MDD and so possibly to offer actually a combined and unified theory which is the one intertwined and open to future investigations.

Extensive literature search indicates that ever since the 1970s, a number of scientific articles studying the potential therapeutic impacts of CBD in different animal models of neuropsychiatric disorders and a few human clinical trials have been published. Since then, knowledge about the new impacts of ECS and CBD and the components of action of these compounds has emerged. New questions showed up with respect to the properties of $\mathrm{CBD}$, and its safety and exact dosage ranges for each disorder. Hence, more randomized, placebo-controlled clinical trials with larger populations will bring critical answers and support the interpretation that supports the translation of them to clinical settings.

\section{Conflict of Interest Statement}

There are no conflicts of interest.

\section{Funding Sources}

The author did not receive any funding.

\section{References}

Role and Function of ECS in MDD
1 Micale V, Ruda-Kucerova J, Drago F, Micale $\mathrm{V}$, Tabiova K. Role of the endocannabinoid system in depression: from preclinical to clinical evidence. 2014

2 Herkenham M, Lynn AB, Johnson MR, Melvin LS, de Costa BR, Rice KC. Characterization and localization of cannabinoid receptors in rat brain: a quantitative in vitro autoradiographic study. J Neurosci. 1991;11(2):563-83.
3 Vinod KY, Hungund BL. Role of the endocannabinoid system in depression and suicide. Trends Pharmacol Sci. 2006;27(10): $539-45$.

4 Russo EB, Burnett A, Hall B, Parker KK. Agonistic properties of cannabidiol at 5-HT1a receptors. Neurochem Res. 2005;30(8):103743. 
5 Linge R, Jiménez-Sánchez L, Campa L, PilarCuéllar F, Vidal R, et al. Cannabidiol induces rapid-acting antidepressant-like effects and enhances cortical 5-HT/glutamate neurotransmission: role of 5-HT1a. Neuropharmacology. 2016;103:16-26.

6 Zanelati TV, Biojone C, Moreira FA, Guimarães FS, Joca SR. Antidepressant like effects of cannabidiol in mice: possible involvement of 5-HT1A receptors. Br J Pharmacol. 2010;159:122-8.

7 Campos AC, Moreira FA, Gomes FV, Del Bel EA, Guimarães FS. Multiple mechanisms involved in the large-spectrum therapeutic potential of cannabidiol in psychiatric disorders. Philos Trans R Soc Lond B Biol Sci. 2012; 367(1607):3364-78.

8 Zanelati TV, Biojone C, Moreira FA, Guimarães FS, Joca SR. Antidepressant like effects of cannabidiol in mice: possible involvement of 5-HT1A receptors. Br J Pharmacol. 2010;159:122-8.

9 Herkenham M, Lynn AB, Johnson MR, Melvin LS, de Costa BR, Rice KC. Characterization and localization of cannabinoid receptors in rat brain: a quantitative in vitro autoradiographic study. J Neurosci. 1991;11(2): 563-83.

10 Herkenham M, Lynn AB, Johnson MR, Melvin LS, de Costa BR, Rice KC. Characterization and localization of cannabinoid receptors in rat brain: a quantitative in vitro autoradiographic study. J Neurosci. 1991;11(2): 563-83.

11 Fanselow MS, Dong HW. Are the dorsal and ventral hippocampus functionally distinct structures? Neuron. 2010;65(1):7-19.
12 Campos AC, Moreira FA, Gomes FV, Del Bel EA, Guimarães FS. Multiple mechanisms involved in the large-spectrum therapeutic potential of cannabidiol in psychiatric disorders. Philos Trans R Soc Lond B Biol Sci. 2012; 367(1607):3364-78.

13 di Giacomo V, Chiavaroli A, Recinella L, Orlando G, Cataldi A, Rapino M, et al. Antioxidant and neuroprotective effects induced by cannabidiol and cannabigerol in rat CTXTNA2 astrocytes and isolated cortexes. Int J Mol Sci. 2020;21(10):3575.

14 Niederhoffer N, Hansen HH, Fernandez-Ruiz JJ, Szabo B. Effects of cannabinoids on adrenaline release from adrenal medullary cells. $\mathrm{Br}$ J Pharmacol. 2001;134(6):1319-27.

15 Hernangómez M, Mestre L, Correa FG, Loría F, Mecha M, Iñigo PM, et al. CD200-CD200R1 interaction contributes to neuroprotective effects of anandamide on experimentally induced inflammation. Glia. 2012;60(9):143750.

16 Campos AC, Fogaça MV, Sonego AB, Guimarães FS. Cannabidiol, neuroprotection and neuropsychiatric disorders. Pharmacol Res. 2016 Oct;112:119-27.

17 Fogaça MV, Reis FM, Campos AC, Guimarães FS. Effects of intra-prelimbic prefrontal cortex injection of cannabidiol on anxietylike behavior: involvement of 5HT1A receptors and previous stressful experience. Eur Neuropsychopharmacol. 2014;24(3):410.

18 Russo EB, Burnett A, Hall B, Parker KK. Agonistic properties of cannabidiol at 5-HT1a receptors. Neurochem Res. 2005;30(8):103743.

19 Niederhoffer N, Hansen HH, Fernandez-Ruiz JJ, Szabo B. Effects of cannabinoids on adrenaline release from adrenal medullary cells. $\mathrm{Br}$ J Pharmacol. 2001;134(6):1319-27.
20 Devinsky O, Nabbout R, Miller I, Laux L, Zolnowska $M$, Wright $S$, et al. Long-term cannabidiol treatment in patients with Dravet syndrome: an open-label extension trial. Epilepsia. 2019 Feb;60(2):294-302.

21 Devinsky O, Cilio MR, Cross H, FernandezRuiz J, French J, Hill C, et al. Cannabidiol: pharmacology and potential therapeutic role in epilepsy and other neuropsychiatric disorders. Epilepsia. 2014;55(6):791-802.

22 Barden N. Implication of the hypothalamicpituitary-adrenal axis in the physiopathology of depression. J Psychiatry Neurosci. 2004; 29(3):185-93.

23 Liu Y, Ho RC, Mak A. Interleukin (IL)-6, tumour necrosis factor alpha (TNF- $\alpha$ ) and soluble interleukin-2 receptors (sIL-2R) are elevated in patients with major depressive disorder: a meta-analysis and meta-regression. J Affect Disord. 2012 Aug;139(3):230-9.

24 Copeland WE, Shanahan L, Worthman C, Angold A, Costello EJ. Cumulative depression episodes predict later C-reactive protein levels: a prospective analysis. Biol Psychiatry. 2012 Jan 1;71(1):15-21.

25 Calabrese F, Rossetti AC, Racagni G, Gass P, Riva MA, Molteni R. Brain-derived neurotrophic factor: a bridge between inflammation and neuroplasticity. Front Cell Neurosci. 2014;8:430.

26 Nagarkatti P, Pandey R, Rieder SA, Hegde VL, Nagarkatti M. Cannabinoids as novel anti-inflammatory drugs. Future Med Chem. 2009; 1(7):1333-49.

27 Onaivi ES, Green MR, Martin BR. Pharmacological characterization of cannabinoids in the elevated plus maze. J Pharmacol Exp Ther. 1990;253(3):1002-9. 